\title{
miR-93-5p Suppresses Ovarian Cancer Malignancy and Negatively Regulate CCND2 By Binding To Its 3'UTR Region
}

\section{Guotong Chen}

Affiliated Hospital of Guangdong Medical University

\section{Yiwei Yan}

Affiliated Hospital of Guangdong Medical University

\section{Xiaojv Qiu}

Affiliated Hospital of Guangdong Medical University

\section{Chengfeng Ye}

Affiliated Hospital of Guangdong Medical University

\section{Xingmei Jiang}

Graduate School of Guangdong Medical University

\section{Shuo Song}

Affiliated Hospital of Guangdong Medical University

Yibo Zhang

Jinan University

\section{Huanhuan Chang}

Affiliated Hospital of Guangdong Medical University

\section{Leqi Wang}

Affiliated Hospital of Guangdong Medical University

\section{Xuehuan He}

Affiliated Hospital of Guangdong Medical University

\section{Lingrong Tang}

Affiliated Hospital of Guangdong Medical University

Qingyu Zhang ( $\sim$ Qingyuzhang@gdmu.edu.cn )

Affiliated Hospital of Guangdong Medical University

\section{Ying Zhang}

Affiliated Hospital of Guangdong Medical University

\section{Research Article}

Keywords: miR-93-5p, ovarian cancer, CCND2, 3'UTR 
Posted Date: November 18th, 2021

DOI: https://doi.org/10.21203/rs.3.rs-1073707/v1

License: (9) This work is licensed under a Creative Commons Attribution 4.0 International License. Read Full License

Version of Record: A version of this preprint was published at Discover Oncology on March 20th, 2022. See the published version at https://doi.org/10.1007/s12672-022-00478-1. 


\section{Abstract}

Ovarian cancer is the most lethal gynecological cancer worldwide, but the underlying mechanism of ovarian cancer malignancy acquirement is largely unknown. miRNA is ubiquitously implicated in disease especially in cancer initiation and progression. In current study, we firstly detected the expression level of miR-93-5p in ovarian cancer patient samples and conducted a survival analysis. Our data revealed miR93-5p is a favorable prognostic factor but is downregulated in ovarian cancer patients. Secondly, CCK8 assay wound healing assay and flow cytometry-based cell cycle analysis and apoptotic cell analysis were performed respectively to study the function of miR-93-5p. Functional analysis show miR-93-5p promotes ovarian cancer malignancy in term of cell proliferation, migration but reduce cell death. Bioinformatic analysis showed Cyclin-D2(CCND2) is a candidate gene of miR-93-5p with the binding site in its $3^{\prime} U T R$ region. Furthermore, quantitive-PCR and western blot were utilized to measure miR-93-5p, CCND2 levels in tissues samples and cell lines. Our data suggested miR-93-5p is negatively correlated to the level of CCND2 mRNA and protein. Finally, Luciferase report assay was conducted, and we demonstrated miR-93-5p reduces CCND2 expression by binding to the 3'UTR region. Our study revealed the function of miR-93-5p in ovarian cancer malignancy and declaimed CCND2 as a target of miR-93-5p.

\section{Introduction}

Ovarian cancer is the most lethal gynecological disease. It is estimated that there are about 240,000 new cases worldwide every year, which account for about $2.5 \%$ of total cancer cases every year but lead to over 150,000 cases of death toll every year[1]. The early symptom of ovarian cancer is not significant and always confused with intestinal disease. Early screen by transvaginal ultrasound (TVUS) and the CA-125 blood test but the TVUS only for a big mass of tumor while CA125 specificity is low[2]. Therefore, $75 \%$ of patients are diagnosed at an advanced stage, and chemotherapy if not become the only choice at least is an irreplaceable intervention option[3, 4] .

miRNA is a class of single-stranded non-coding ribonucleotides with a length of approximately 22 nucleotides, which is always transcript by a complete endogenous gene and functions as gene expression regulators of parental genes[5]. It is predicted over $60 \%$ of human genes are regulated by miRNA through binding to the 3'untranslated region (3'UTR) or coding region of the target gene mRNA[6]. MicroRNA can simultaneously regulate multiple target genes which leads to cell adaptive change to the microenvironment. It has been reported that microRNA is implicated in cancer progression in various of cancer for example miR-135a-3p, miR-200c, miR-216a, and miR-340[7, 8]. miRNA could be promising target for cancer early diagnosis and prognosis.

In this study, we found that the expression of miR-93-5p in cancer tissues is lower than that in normal tissues. The target binding prediction suggests that CCND2 may be the target gene of miR-93-5p. Artificially transfection miR-93-5p mimics to ovarian cancer cells can downregulate the CCND2 gene expression and its protein levels. Cell function assay confirms miR-93-5p is a negative regulator for malignancy of ovarian cancer cells such as migration, proliferation, and survival. The data from the 
clinical sample suggested miRNA -93-5p has a negative correlation with CCND2 levels in cancer patients. Our results identified that miR-93-5p are functional downregulated in ovarian cancer which can inhibit ovarian cancer malignancy by regulating CCND2.

\section{Materials And Methods}

\section{Patient and Tissue Samples}

Human ovarian cancer and normal ovarian tissues Samples were obtained from the Affiliated Hospital of Guangdong Medical University(Guangdong, China)From January 2019 to June 2020. This research was approved and supervised by the Hospital Ethics Committee of the Affiliated Hospital of Guangdong Medical University. All patients have signed the informed consent to agree to the use of tissue samples for research purposes. All patients had not received radiotherapy or chemotherapy before surgery, and the pathology diagnosis of the samples was determined according to postoperative tissue immunohistochemistry.

\section{Cell Culture}

Human epithelial ovarian cancer cell line A2780 was obtained from the Shanghai Gaining Biological Ltd. This cell line was cultured in RPMI-1640 supplemented with 10\% fetal bovine serum (FBS). Human normal ovarian epithelial cell line IOSE80 was obtained from the BeNa Culture Collection Ltd. This cell line was cultured in DMEM supplemented with $10 \%$ fetal bovine serum (FBS). All the cell lines were maintained at $37^{\circ} \mathrm{C}$ in a $5 \% \mathrm{CO} 2$ humidified atmosphere.

\section{RNA Extraction, cDNA Synthesis and qPCR}

Total RNA was extracted from tissues or cells using Trizol(Takara, Japan)and cDNA prepared and subsequently quantitated using Mir- $\mathrm{X}^{\mathrm{TM}}$ miRNA First-Strand Synthesis and TB Green ${ }^{\circledR}$ qRT-PCR(Takara, Japan $\rrbracket$ performed on ABI 7500 qRT-PCR System U6 was used as the internal control for quantitative PCR of miRNA, while the beta-actin was used as internal control used for quantitative PCR of CCND2. The primer used in this study is listed below. 
Table 1

Primer sequences used for $\mathrm{QPCR}$

\begin{tabular}{|ll|}
\hline Primer name & Sequences $\left(\mathbf{5}^{\prime} \sim \mathbf{3}^{\prime}\right.$ ) \\
\hline miR-93-5p & F: GCCGCCAAAGTGCTGTTC \\
\cline { 2 - 2 } U6 & R: CAGAGC AGGGTCCGAGGTA \\
& F: CTCGCTTCGGCAGCACA \\
\hline R: AACGCTTCACGAATTTGCGT \\
ACTIN & F: TTGTGATGCCCTGACTGAGC \\
& R: CACGTTGGTCCTGACGGTACT \\
\cline { 2 - 2 } & F: AGCGAGCATCCCCCAAAGTT \\
\hline & R: GGGCACGAAGGCTCATCATT \\
\hline
\end{tabular}

\section{Western Blot}

Cells or tissues proteins were extracted using precooled RIPA lysis buffer. Sample lysed and centrifugate for $5 \mathrm{~min}$ at $12,000 \mathrm{rpm}, 4^{\circ} \mathrm{C}$. Collect the protein supernatant and detect the protein concentration, then add loading buffer to prepare the protein sample for the WB experiment. Proteins were separated by $10 \%$ SDS-PAGE electrophoresis and transferred to polyvinylidene difluoride (PVDF) membranes. Membranes were blocked with $5 \%$ no-fat milk for $1 \mathrm{~h}$, then cut the required protein bands(CCND2 $31 \mathrm{kDa}$, a-Tubulin 52 $\mathrm{kDa}$ )and incubated with primary antibodies $\left(1: 1000\right.$, obtained from CST) at $4^{\circ} \mathrm{C}$ overnight. Membranes were washed with TBST and incubated for $1 \mathrm{~h}$ with secondary antibodies $(1: 1,000$, obtained from CST) at room temperature. Enhanced chemiluminescence (ECL) kit and Canon 5200 were used to visualize the protein signal.

\section{Construction and Transfection of miRNA}

The miR-NC and miR-93-5p mimic were purchased from Shanghai GenePharma Co., Ltd (Shanghai, China). miRNA were transfected with Lipofectamine 3000(Invitrogen, Carlsbad, CA, USA)when the density of the cell inoculation plate is about $50 \%$.

\section{CCK8 assay}

A total of 5000 A2780 cells were pre-seeded in each well of the 96 -well plates. the cells were transfected with miRNA when cells confluence upto $80 \%$. after $0 \mathrm{~h}, 24 \mathrm{~h}, 48 \mathrm{~h}$, and $72 \mathrm{~h}$ transfection, $10 \mu \mathrm{l}$ of CCK 8 solution(Yeasen, China) was added to each well and incubated for 1 hour and read the absorbance at $450 \mathrm{~nm}$ by a microplate reader.

\section{Cell Cycle Analysis}

To analyze the cell cycle distribution, A2780 cells were plated in 12-well plates at a density of $10 \times 104$ cells per well, transfected after the cells were adhered to the wall. 48 hours after transfection, the cells 
were digested with trypsin. Washed and centrifuged with precooled PBS then discarded the supernatant. $1 \mathrm{ml} 70 \%$ ethanol was added into the centrifuge tube to resuspend cells and fix the cell at $4{ }^{\circ} \mathrm{C}$ for $24 \mathrm{hrs}$. After fixation, cells were washed with PBS and centrifuged, discarded the supernatant, and add the configured PI (Beyotime Biotechnology, China) to avoid light staining for 30 minutes, and then detect by flow cytometry.

\section{Wound healing Assay}

A total of $5 \times 10^{5} \mathrm{~A} 2780$ cells were seeded in each well of the 6 -well plates, transfected after the cells were adhered to the wall. After 24 hours of transfection, cleaned the cell culture plate twice with PBS, then scratch from top to bottom with the end of a sterile pipette tip. Rinsed gently with PBS twice and then added an appropriate amount of $2 \%$ fetal bovine serum culture medium. Took pictures of cell scratches in 0 and 48 hours, then analyzed cell scratch healing rate by Image $\mathrm{J}$.

\section{Apoptosis Analysis}

The A2780 cells were seeded in 12-well plates at a density of $10 \times 10^{4}$ cells per well overnight and cells were transfected with miRNA. after 48 hours of transfection, the cells were digested with trypsin and collected in the Eppendorf tube. Washed and centrifuged with precooled PBS then discarded the supernatant 2 times. Annexin V FITC Apoptosis kit(BD, American) was used for apoptotic cell analysis. The cells were resuspended in 500 $\mu$ l staining buffer and stained with $10 \mu$ of each Annexin V FITC and PI for 15 mins protecting from light at room temperature. The well-stained cells were analyzed by flow cytometry.

\section{Luciferase report assay}

In order to verify the target between CCND2 and miR-93-5p, the CCND2 wild(CCND2-wt) and mutant CCND2(CCND2-mut) vectors were synthesized by CCND2 cDNA fragment insertion which contains the wild or mutant binding site of miR-93-5p into the pmirGLO dual-luciferase miRNA target expression vectors. Furthermore, 293T cells were co-transfected with pmirGLO dual-luciferase reporter vectors (CCND2-wt and CCND2-mut) and miR-93-5p mimics or negative control. After 48 hours, cells were harvested, and the luciferase activity was measured using the dual-luciferase assay system (Beyotime Biotechnology, China) following the instruction of manufacture, relative to that renilla luciferase.

\section{Immunohistochemistry}

The collected specimens were fixed in $4 \%$ paraformaldehyde for at least $72 \mathrm{hrs}$ and then dehydrated within an automatic dehydrator. Samples were embedded in liquid paraffin and store at $4^{\circ} \mathrm{C}$ overnight. The tissues were then sliced $3 \mu \mathrm{m}$ thick. The specimens were heated at $60^{\circ} \mathrm{C}$ for $2 \mathrm{hrs}$ and then deparaffinized in xylene and rehydrated in a series of graded alcohol. The sample was pretreated in 0.01 $\mathrm{M}$ Citrate buffer and make a heat-induced antigen retrieval was carried out by using the microwave to heat buffer to boil for $15 \mathrm{~min}$. The slices were then incubated in $3 \% \mathrm{H}_{2} \mathrm{O}_{2}$ and $0.1 \%$ Tween 20 at room temperature without light for 20 min to block endogenous peroxidase activity. Then slices were blocked by $5 \%$ goat serum for $30 \mathrm{~min}$. Afterward, a primary antibody was incubated at $4^{\circ} \mathrm{C}$ overnight. 
Subsequently, they were incubated with a secondary antibody for 1 h. 3,3》- diaminobenzidine (DAB) chromogen was applied and then stained with hematoxylin. The slices then were dehydrated through graded alcohol and cleared in xylene and photographed under a microscope

\section{Statistical Analysis}

The result was expressed as mean \pm standard error. Test the normality and variance equivalence of all data to determine the appropriate statistical test. A nonparametric test or independent sample T-test was used to determine significant differences in the group. The data were analyzed using Prism 8 (GraphPad, La Jolla, CA, USA). $\mathrm{P}<0.05$ was considered statistical significance.

\section{Results}

\section{1. miRNA-93-5p is a positive prognosis marker but was downregulated in ovarian cancer}

First, we measured the miR-93-5p level in ovarian cancer tumor tissues using qPCR assay. The tumor tissue RNA was extracted by Trizol and cDNA was synthesized by a first-strand cDNA synthesis kit from Takara. Specific primer was used for qPCR and the melt curve was confirmed the primer is specific to the miR-93-5p. 2-delta delta CT was used to calculate the miR-93-5p level in each sample. The result shows the miR-93-5p level in tumor cells is dramatically decreased (Fig.1A). meanwhile, we found that CCND2, a cell cycle master, was significantly increased in ovarian cancer tumor tissues accordingly. To confirm the pattern of CCND2 in tumor tissues, we further analyzed CCND2 levels in tumor tissues by both western blot and IHC assay. Result clear show CCND2 level in tumor tissues $(n=12)$ are higher than non-tumor ovary tissues( $n=15)$ (Fig.1C). To investigate the relationship between the miR-93-5p and CCND2 in tumor tissues, we first make a correlation analysis and the result suggest that miRNA-93-5p is negatively correlated with mRNA level of CCND2 ( $n=6, R=-0.467, p=0.134$, Fig.1D). To explore the role of miR-93-5p in ovarian cancer progression, we utilized the data from the project of The Cancer Genome Atlas and make the survival curve of patients with the different expression levels of miR-93-5p. The result clearly shows that low expression of miR-93-5p is a poor prognosis factor of patient survival time $(n=200, p<0.01)$.

\section{2. miR-93-5p suppresses ovarian cancer cell malignancy by negative regulation of CCND2}

To investigate the function of miR-93-5p in ovarian cancer cell malignancy, we transfection of miR-93-5p mimics to A2780 cell, in which CCND2 was upregulated. After miR-93-5p transfection for $24 \mathrm{~h}$, cell morphology was observed under a phase-contrast microscope (Fig.2A) and a CCK8 assay was performed to verify the cell proliferation. The result showed that miRNA-NC did not affect cell viability compared to the transfection reagent control group. But miR-93-5p mimic transfection can significantly reduce cell viability in a time-dependent manner (24h, 48h, 72h $p<0.01$, Fig. 2B). Therefore, we wonder if miR-93-5p caused cell proliferation reduction result from cell cycle arrest. We test the cell cycle in the miR-93-5p and miR-NC group by PI staining of DNA and flow cytometry. The result showed miR-93-5p transfection 
increases $\mathrm{G} 1$ phase cell and reduces $S$ phase cell, which suggests that miR-93-5p could inhibit cell proliferation by blocking the cell cycle at G1/S transition. We observed cell death increase and we wonder whether miR-93-5p can induce cell apoptosis and result indicated that miR-93-5p increases cell apoptosis up to 3-folds compared to the miR-NC group (Fig.2E). We further test miR-93-5p function on cell migration, wound healing assay was performed, and the result showed miR-93-5p also inhibits cell migration but is not significant to the cell proliferation and cell apoptosis (Fig.2D).

\section{3. miR-93-5p negatively regulates CCND2}

To answer why miR-93-5p suppresses ovarian cancer cell proliferation and cell cycle arrest, we screened the potential target of miR-93-5p in three miRNA public databases including TargetScan, miRGate, miRWalk. We found out 5 potential targets in the three databases by Venn plot analysis. The 5 candidates are listed on the right of Fig.3A and CCND2 is a well-known oncogenic gene involved in cell cycle G1/S transition. We predicate the binding site of miR-93-5p using the RNA22v2 tool (https://cm.jefferson.edu/rna22/Interactive/) and the result indicated miR-93-5p can bind the 3'UTR of CCND2 mRNA (Fig.3B). we detect CCND1 in ovarian cancer cell A2780 and immortal ovarian epithelial cell IOSE8 and we found CCND2 in A2780 cell is higher than in IOSE8 (Fig.3C). Thereby, we quantify the miR-93-5p level in both cell lines by qPCR. The result suggests the miR-93-5p level in A2780 is lower than the IOSE8 cell line (Fig.3D). Transfection of miR-93-5p mimics into A2780 cells can significantly reduce the CCND2 protein level (Fig.3E). To confirm miR-93-5p can regulate CCND2, CCND2 3'UTR was constructed into pmirGLO Dual-Luciferase miRNA Target Expression Vector. A mutant of 3'UTR was also constructed into the vector as a control. After transfection of miR-93-5p mimic or miR-NC, the luciferase activity was detected, and results show miR-93-5p only can significantly reduce firefly luciferase in the 3'UTR of WT CCND2 mRNA (Fig.3F).

\section{Discussion}

Since the first miRNA was discovered in 1993, the understanding of miRNA biology in development and disease has greatly increased, especially in many cancer cases, which affect the cell cycle, differentiation, apoptosis, migration and drug resistance so on and so forth [9]. As a regulatory molecule for homologous genes, it is predicted that more than $60 \%$ of human protein-coding gene mRNA dynamic level is regulated by miRNA [6]. Although miRNA is small, it is more stable than mRNA. It is highly stable in blood, urine and other body fluids. The detection of miRNA levels in corresponding body fluids has the potential for early cancer diagnosis and predicting the prognosis of treatment. MiRNA could serve as a novel of biomarker for cancer diagnosis and prognosis $[10,11]$. Our research found that miR-93-5p is low expressed in ovarian cancer and is related to prognosis. However, due to its low expression, combining with tumorrelated indicators such as CA125, CA199 would help improve clinical tools in diagnosis and prognosis of ovarian cancer.

We found that the expression of miR-93-5p is significantly down-regulated in ovarian cancer and is a protective factor for the survival of ovarian cancer patients, and functional experiments indicate that miR93-5p can significantly inhibit the malignant characteristics of ovarian cancer. It is reported that miR-93- 
$5 p$ has a differential table in a variety of malignant tumors, especially in digestive system tumors. The low expression of miR-93 in colon cancer is closely related to its metastasis, differentiation and poor prognosis. Up-regulation of miR-93 expression can inhibit the metastasis and invasion of colon cancer by inhibiting the Wnt / $\beta$-catenin signaling pathway [12]. In liver cancer, miR-93 can negatively regulate the expression of the tumor suppressor gene PTEN, and promote the proliferation, invasion and migration of hepatocellular carcinoma cells by activating the c-Met / PI3K / Akt signaling pathway [13]. In gastric cancer, miR-93 negatively regulates the tumor suppressor gene Programmed cell death 4 (PDCD4), thereby promoting the development of gastric cancer [14]. It is important to note that compared with precancerous lesions and normal tissues, ovarian cancer has low expression of miR-93, and the expression level of miR-93 is negatively correlated with the differentiation grade of ovarian cancer and the FIGO stage of ovarian cancer. [15].Therefore, it is suggested that miR-93-5p may be a tumor-related miRNA implicated in various of tumor type.

After screening the target pool of miRNA through miRWalk, miRGate, TargetScan analysis, we found out CCND2 could be a candidate target gene of miR-93-5p. CCND2 is a member of the cyclin family and is a key regulator of the mammalian cell cycle and always upregulated in carcinogenesis of tumors [16]. The complex formed by CCND2 and CDK4/6 (CCND2-CDK4/6) regulates the phosphorylation and inactivation of tumor suppressor retinoblastoma protein (RB) to prompt cells transition from $\mathrm{G} 1$ phase to $S$ phase [17]. CCND2 upregulated and could be a therapeutic target for a variety of tumors, including colon cancer, stomach cancer, liver cancer, breast cancer and lung cancer, etc. [18-21]. We found that the expression of CCND2 in epithelial ovarian cancer tissue and epithelial ovarian cancer cell A2780 was higher than that of normal ovarian tissue and normal epithelial ovarian cancer cell IOSE80, respectively. miR-93-5p was overexpressed in epithelial ovarian cancer cell A2780. The CCK8 assay showed that the cell proliferation ability decreased and the flow cytometry showed that the proportion of cells in the G1 phase of the cell cycle increased indicated miR-93-5p suppress cell cycle $S$ phase transition which may answer the role of miR-93-5p inhibition of cell proliferation. Interestingly, transfection of miR-93-5p mimic into ovarian cancer cell line A2780 can negatively regulate the expression of CCND2 mRNA and protein level. Luciferase reporter assay showed miR93-5p can partly reduce CCND2 by binding its 3'UTR. We found cell migration and apoptosis did not too much significantly affect miR-93-5p than in terms of cell proliferation which may result from miR-93-5p promoting degradation mRNA of CCND2. Finally, we confirmed that miR-93-5p and CCND2 expression is negatively correlated in tumor tissues of patients with ovarian cancer. These data indicate that upregulation of CCND2 by reduction of miR-93-5p probably is conducive to ovarian cancer tumor growth and survival. Our finding partly revealed the mechanism of miR-93-5p in the evolution of ovarian cancer cells and its clinical potential in the battle against ovarian cancer.

\section{Declarations}

\section{Acknowledgement}

We appreciate the guidance and advice of Dr. Xilan Shi in polishing of manuscript. 


\section{Authors' contributions}

QYZ wrote the manuscript, GTC, YWY, XJQ, XMJ and did the experiment and made diagrams; SS, YBZ, CFY, HHC, LQW, XHH, LRT contributed substantial advice help to polish the language. YZ, QYZ conducted the project and revised the whole manuscript.

\section{Funding}

This work was supported by the Natural Science Foundation of Guangdong Province (2017A030313559) and Zhanjiang science and Technology Bureau (NO. 2020A100302). The National Natural Science Foundation of China (NO.81902801)

\section{Availability of data and materials}

The authors confirm that the data supporting the findings of this study are available within the article

\section{Ethics approval and consent to participate}

This research was approved and supervised by the Hospital Ethics Committee of the Affiliated Hospital of Guangdong Medical University; all patients signed informed consent.

\section{Consent for publication}

All authors consent to publication.

\section{Competing interests}

The authors declare that they have no competing interests.

\section{References}

1. Sung H, Ferlay J, Siegel RL, Laversanne M, Soerjomataram I, Jemal A, et al. Global Cancer Statistics 2020: GLOBOCAN Estimates of Incidence and Mortality Worldwide for 36 Cancers in 185 Countries. CA: a cancer journal for clinicians. 2021; 71: 209-49.

2. Nash Z, Menon U. Ovarian cancer screening: Current status and future directions. Best Pract Res Clin Obstet Gynaecol. 2020; 65: 32-45.

3. Lheureux S, Gourley C, Vergote I, Oza AM. Epithelial ovarian cancer. Lancet. 2019; 393: 1240-53.

4. Vergote I, Denys H, De Greve J, Gennigens C, Van De Vijver K, Kerger J, et al. Treatment algorithm in patients with ovarian cancer. Facts Views Vis Obgyn. 2020; 12: 227-39.

5. O'Brien J, Hayder H, Zayed Y, Peng C. Overview of MicroRNA Biogenesis, Mechanisms of Actions, and Circulation. Frontiers in Endocrinology. 2018; 9.

6. Friedman RC, Farh KK, Burge CB, Bartel DP. Most mammalian mRNAs are conserved targets of microRNAs. Genome Res. 2009; 19: 92-105. 
7. Ghafouri-Fard S, Shoorei H, Taheri M. miRNA profile in ovarian cancer. Exp Mol Pathol. 2020; 113: 104381.

8. Peng Y, Croce CM. The role of MicroRNAs in human cancer. Signal Transduction and Targeted Therapy. 2016; 1: 15004.

9. Lee YS, Dutta A. MicroRNAs in cancer. Annu Rev Pathol. 2009; 4: 199-227.

10. Schwarzenbach H, Nishida N, Calin GA, Pantel K. Clinical relevance of circulating cell-free microRNAs in cancer. Nat Rev Clin Oncol. 2014; 11: 145-56.

11. Armand-Labit V, Pradines A. Circulating cell-free microRNAs as clinical cancer biomarkers. Biomol Concepts. 2017; 8: 61-81.

12. Tang Q, Zou Z, Zou C, Zhang Q, Huang R, Guan X, et al. MicroRNA-93 suppress colorectal cancer development via Wnt/ $\beta$-catenin pathway downregulating. Tumour Biol. 2015; 36: 1701-10.

13. Ohta $\mathrm{K}$, Hoshino $\mathrm{H}$, Wang J, Ono S, lida $\mathrm{Y}$, Hata $\mathrm{K}$, et al. MicroRNA-93 activates c-Met/PI3K/Akt pathway activity in hepatocellular carcinoma by directly inhibiting PTEN and CDKN1A. Oncotarget. 2015; 6: 3211-24.

14. Liang $\mathrm{H}$, Wang F, Chu D, Zhang W, Liao Z, Fu Z, et al. miR-93 functions as an oncomiR for the downregulation of PDCD4 in gastric carcinoma. Sci Rep. 2016; 6: 23772.

15. Chen X, Chen S, Xiu YL, Sun KX, Zong ZH, Zhao Y. RhoC is a major target of microRNA-93-5P in epithelial ovarian carcinoma tumorigenesis and progression. Mol Cancer. 2015; 14: 31.

16. Sherr CJ, Beach D, Shapiro GI. Targeting CDK4 and CDK6: From Discovery to Therapy. Cancer Discov. 2016; 6: 353-67.

17. Gao X, Leone GW, Wang H. Cyclin D-CDK4/6 functions in cancer. Adv Cancer Res. 2020; 148: 14769.

18. Park SY, Lee CJ, Choi JH, Kim JH, Kim JW, Kim JY, et al. The JAK2/STAT3/CCND2 Axis promotes colorectal Cancer stem cell persistence and radioresistance. J Exp Clin Cancer Res. 2019; 38: 399.

19. Oshimo Y, Nakayama H, Ito R, Kitadai Y, Yoshida K, Chayama K, et al. Promoter methylation of cyclin D2 gene in gastric carcinoma. Int J Oncol. 2003; 23: 1663-70.

20. Lehmann U, Berg-Ribbe I, Wingen LU, Brakensiek K, Becker T, Klempnauer J, et al. Distinct methylation patterns of benign and malignant liver tumors revealed by quantitative methylation profiling. Clin Cancer Res. 2005; 11: 3654-60.

21. Hung CS, Wang SC, Yen YT, Lee TH, Wen WC, Lin RK. Hypermethylation of CCND2 in Lung and Breast Cancer Is a Potential Biomarker and Drug Target. Int J Mol Sci. 2018; 19.

\section{Figures}


A

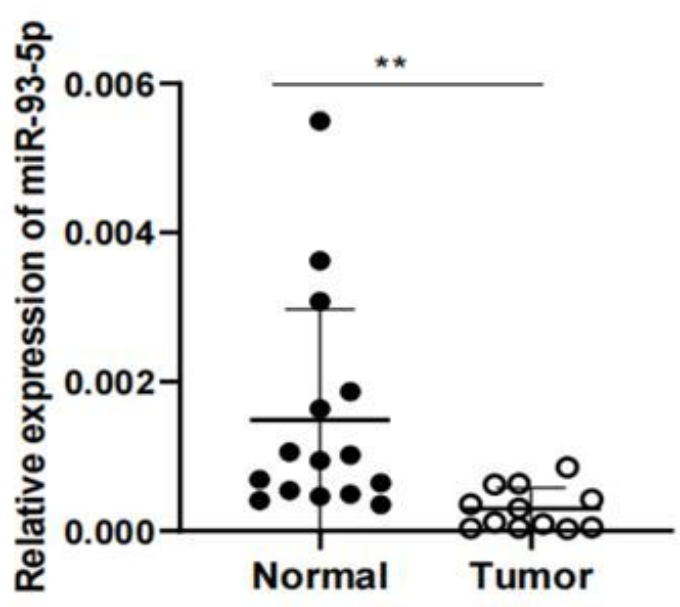

C

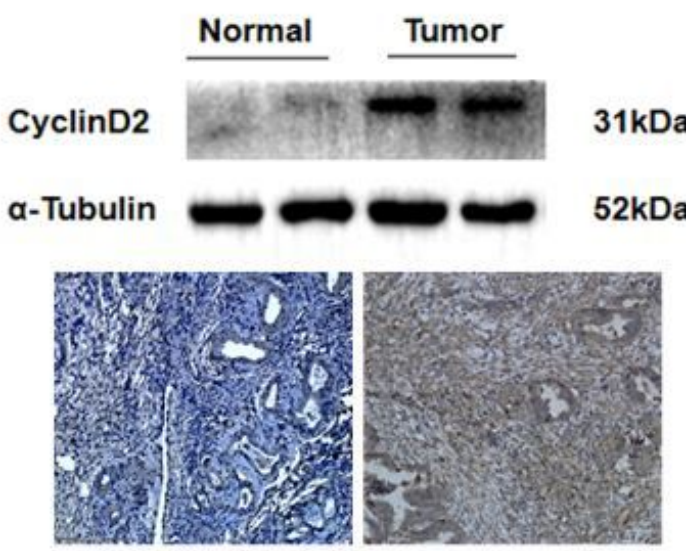

D

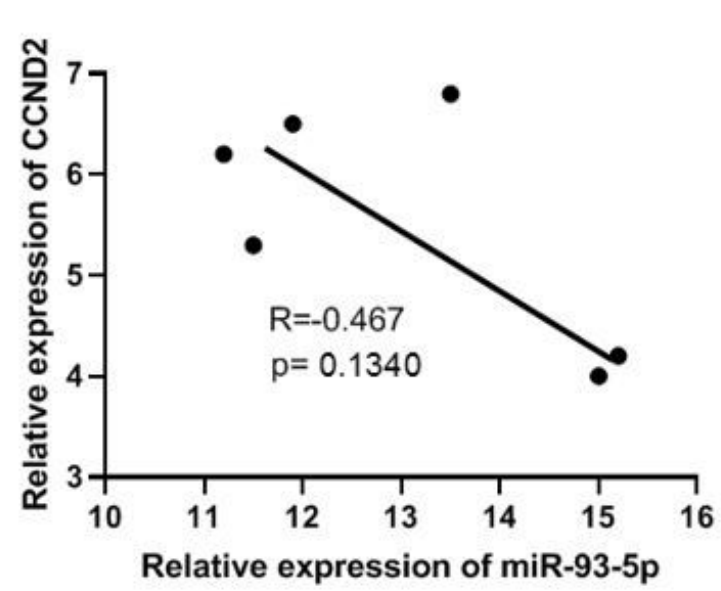

B
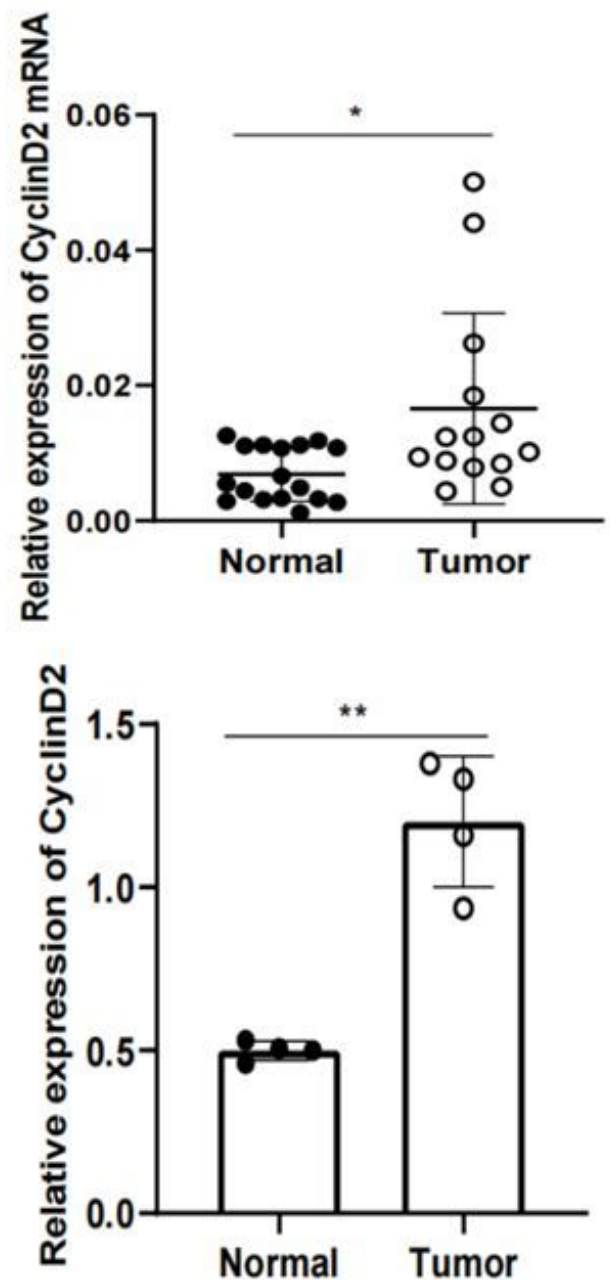

$\mathbf{E}$

The survival of TCGA $(n=200)$

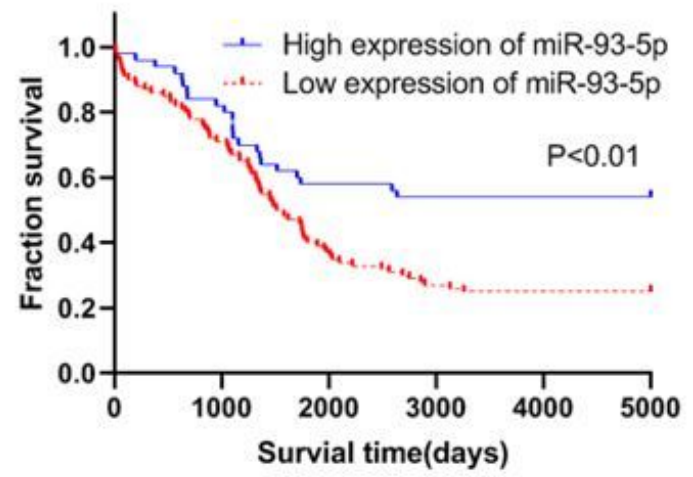

Figure 1

miR-93-5p is downregulated in ovarian cancer and predicate good outcome of patients in term of overall survival A.The qPCR results revealed that the expression of miR-93-5p was low in ovarian cancer group $(\mathrm{P}<0.01)$. B.The qPCR results revealed that the expression of CCND2 was low in ovarian cancer group $(\mathrm{P}<0.01)$. C.CCND2 is highly expressed in human epithelial ovarian cancer. IHC experiment observed that compared with normal ovarian tissue, the staining of CCND2 in ovarian cancer tissue was more 
obvious, and there were more yellow staining granules $₫$ which means high expression in ovarian cancer. Scale bar $=300 \mu \mathrm{m}$. D. The relative expression of miR-93-5p and CCND2 has no significant correlation at the RNA level. $* \mathbb{P}<0.05 \rrbracket * \star \otimes P<0.01$. E. Kaplan-Meier Plotter shows that low expression of miR-93-5p is a risk factor for patients with ovarian cancer.

A

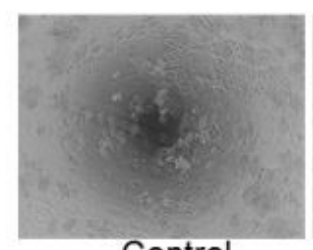

Control

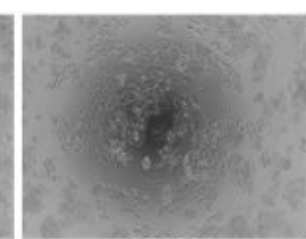

miR-NC

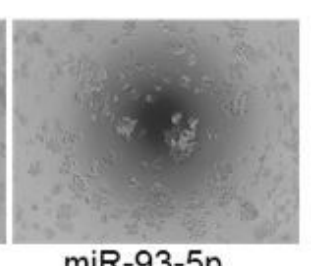

miR-93-5p
B

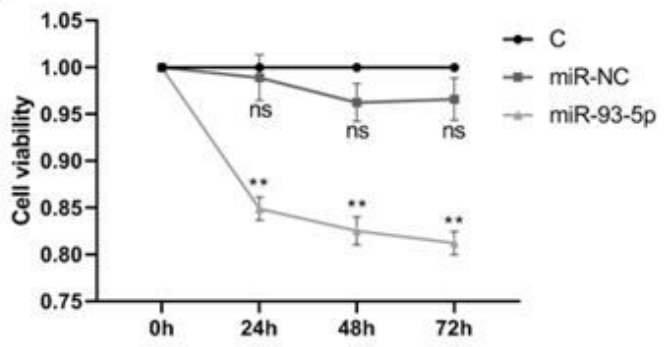

C

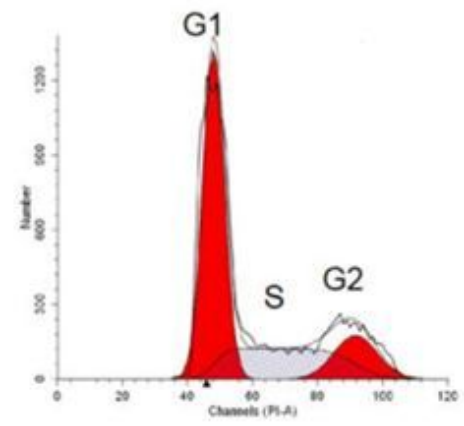

miR-NC

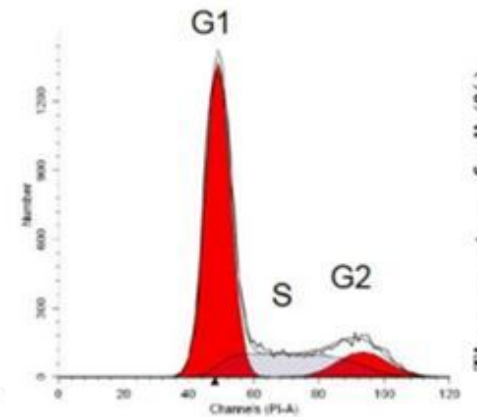

miR-93-5p
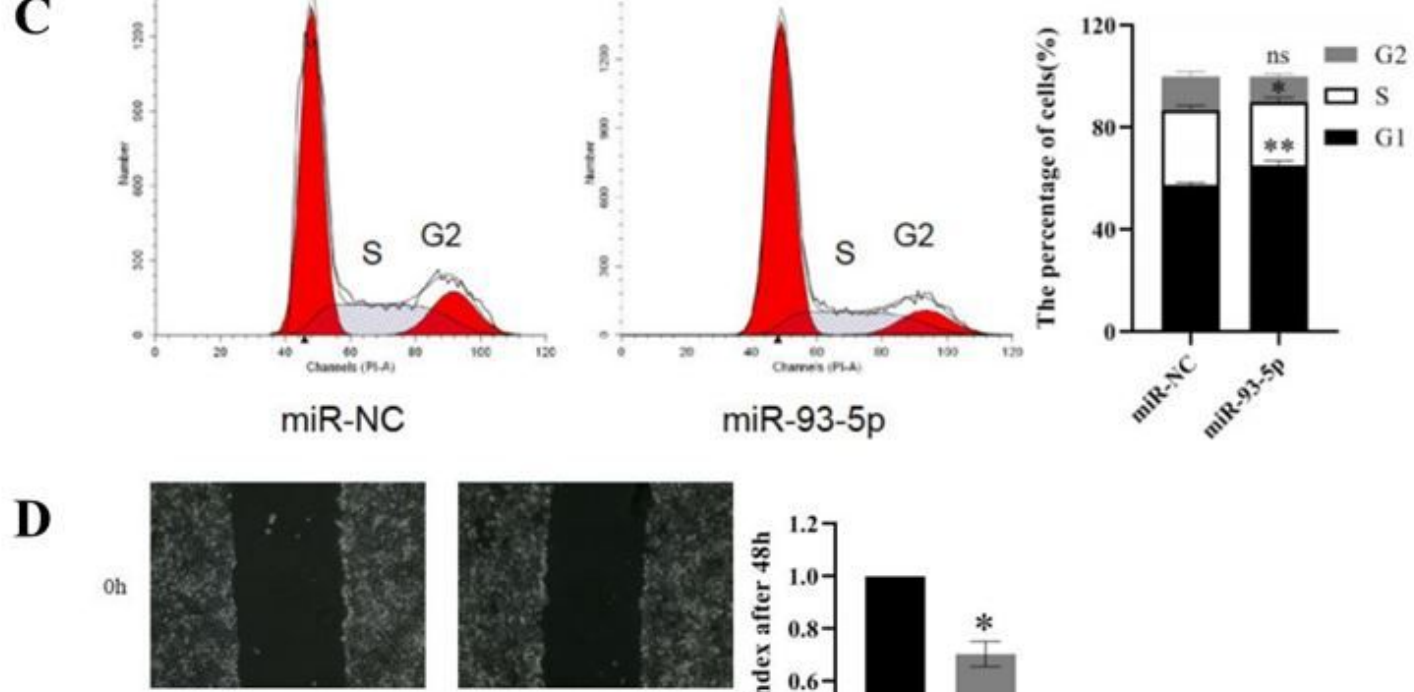

$48 \mathrm{~h}$
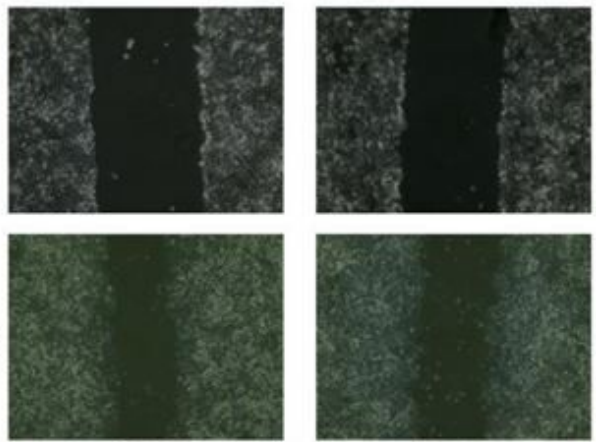

miR-NC
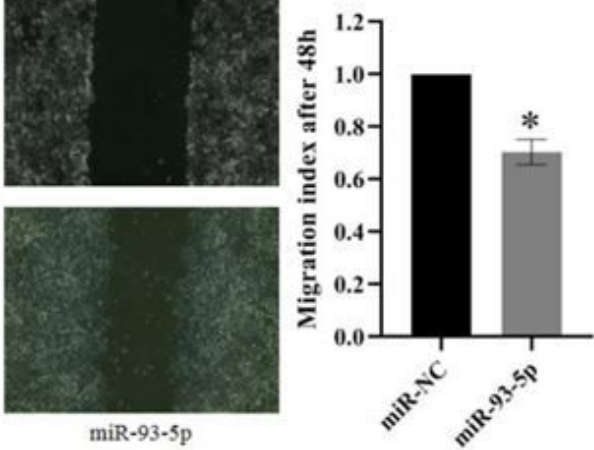

$\mathbf{E}$

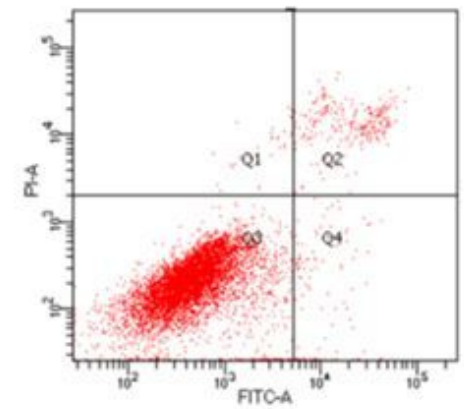

miR-NC

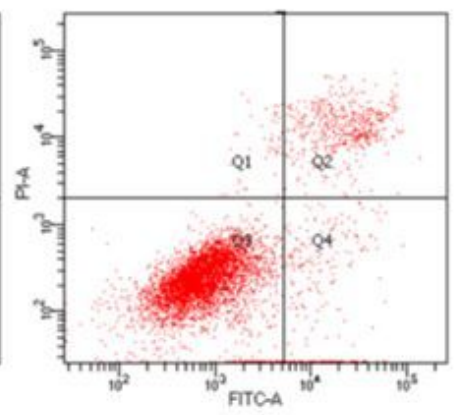

miR-93-5p

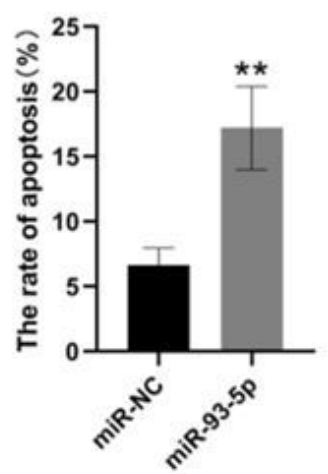

Figure 2 
miR-93-5p suppresses malignancy of ovarian cancer A2780 cells A. The images of A2780 cells under phrase contrast microscope after treatment of either miR-NC or miR-93-5p mimic for 48 hours. B. CCK8 assay was performed to measure cell viability after treatment of miR-93-5p mimic and miR-NC in A2780 cells. C. Cell cycle analysis was performed using PI staining and flow cytometry. Wound healing assay were performed to test the cell migration ability after transfection of miR-93-5p mimic and miR-NC in A2780 cell. E. Cell apoptosis was analyzed by using AnnexinV-FITC/PI kit based on flow cytometry approach after cell transfection of miR-93-5p mimic and miR-NC in A2780 cells. $* \mathbb{P}<0.05 \rrbracket \star \star \otimes P<0.01$

A

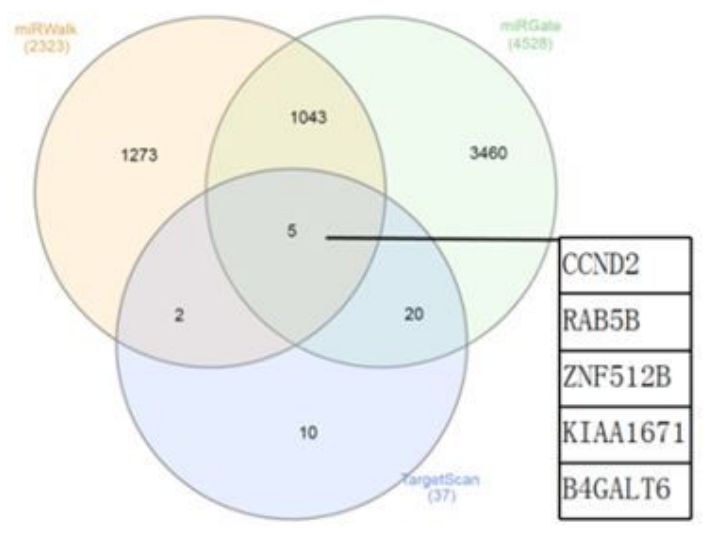

C

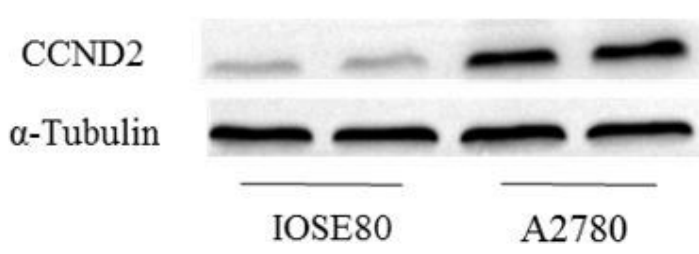

$\mathbf{E}$

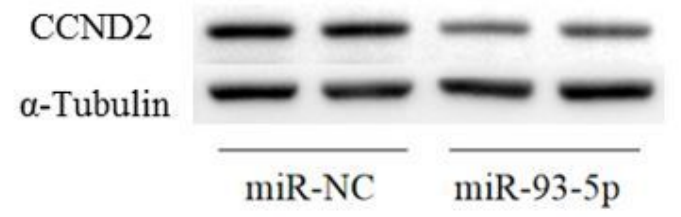

\section{B}

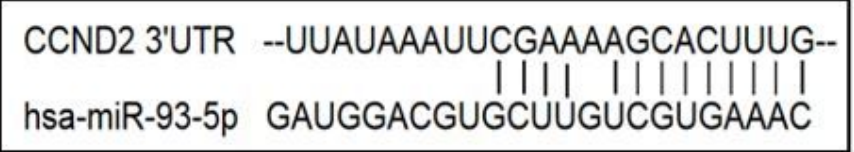

D

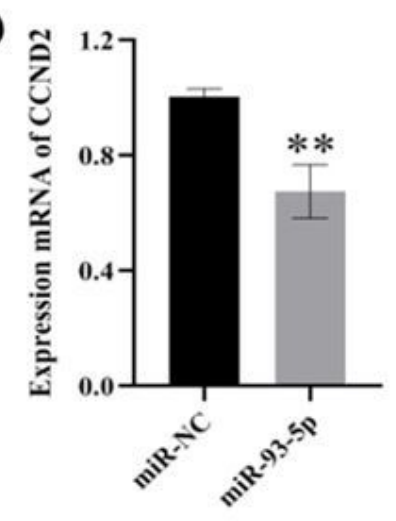

F

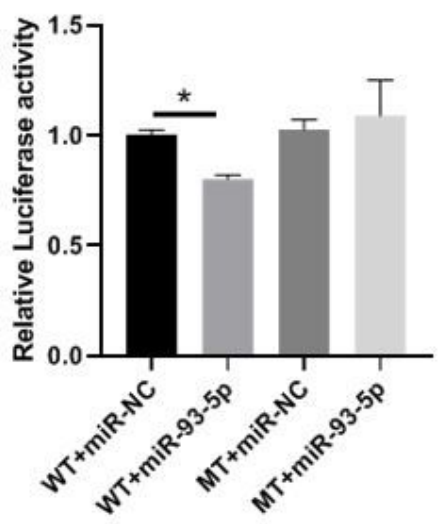

\section{Figure 3}

miR-93-5p reduce CCND2 by binding 3'UTR of CCND2 mRNA A. Using TargetScan $\square m i R G a t e \llbracket m i R W a l k$ and other miRNA databases predicted that CCND2may be the target gene of miR-93-5p. B. Prediction of target binding sites between miR-93-5p and CCND2 by database RNA22v2. C. CCND2 protein level in ovarian 
cancer cell A2780 and ovary immortalized cell IOSE80 was measured by western blot. Tubulin was used as internal control. D. The qPCR assay was performed to detect the mRNA expression of CCND2 after miR-93-5p mimic or miR-NC transfection. Tubulin were used as internal control. ${ }^{*} P<0.01$. E. Western Blot were used to detect the CCND2 protein level after transfection of miR-93-5p mimic or miR-NC. ${ }^{*}<<0.05 \square$ $\star \star P<0.01$. F.The CCND2 gene with wild type binding site or mutant expression level were tested by luciferase reporter assay, ${ }^{*} \mathrm{P}<0.01$. 